\title{
Hip Fracture Management Using External Fixation-A Systematic Review
}

Yu Han $\underline{\text { Chee, }},{ }^{1} M B C h B(U K), M R C S(E d), F R C S(E d)$, Jenies Hui Xin $\underline{\text { Foo }},{ }^{2} M B B S$, Si Jian $\underline{\text { Hui }},{ }^{2} M B B S$, Kuang Ian $\underline{\text { Tan }},{ }^{2} M B B S$

\begin{abstract}
Introduction: Patients with significant comorbidities have high general anaesthetic risks and are often thought to be undesirable candidates for general anaesthesia and, therefore, surgery. External fixation uses local or regional anaesthesia, and allows patients with significant comorbidities to avoid the risks of general anaesthesia. It has been described to be successful in the management of high-risk patients with intertrochanteric fractures. However, published data have been derived from small case series, and no published literature has attempted to analyse them in totality. This review aims to pool together these case series, and to evaluate the outcomes and complications of external fixation when performed in high-risk patients with intertrochanteric fractures. Materials and Methods: The review was conducted using the Preferred Reporting Items for Systematic Reviews and Meta-Analyses (PRSIMA) guidelines. All studies that reported the outcomes of external fixation for intertrochanteric fractures of high-risk patients were included. Results: A total of 13 publications, involving 687 patients, were included in the review. All the studies reported postoperative radiological reduction and complete fracture healing with reduction of limb length discrepancy. One study using parallel placement of proximal fixation screws showed shorter operative duration as compared to convergent placement. Another study mentioned that there was no significant difference in mortality rates between patients with stable fractures and those with unstable fractures who underwent external fixation. All the studies reported a decrease in postoperative immobility, reduction in pain and improvement in clinical outcome hip scores. Conclusion: External fixation is promising and useful especially in the management of high-risk patients with intertrochanteric fractures. The procedure can help with radiological reduction of the fracture, reduction of limb length discrepancy, reduction of operative duration, decrease in postoperative immobility, reduction in pain and improvement in clinical outcome hip scores. The procedure is versatile and seems to be able to accommodate both stable and unstable fractures. However, unstable fractures may be associated with greater postoperative morbidity, and it may be worthwhile to prognosticate based on the stability of the patients' fracture for better risk-benefit analysis preoperatively. Shorter operative times can also be achieved through parallel proximal pin placement, without impact on mortality or morbidity.
\end{abstract}

Ann Acad Med Singapore 2020;49:477-88

Key words: External fixation; High risk; Intertrochanteric

\section{Introduction}

Hip fractures are a common problem in the elderly. In Singapore, the hip fracture incidence in men and women has risen 1.5 times and 5 times over a span of 3 decades respectively, and the age-adjusted rates among women over the age of 50 years old are currently among the highest in Asia. The incidence of hip fractures per annum is projected to increase from

'Division of Musculoskeletal Trauma Surgery, Department of University Orthopaedics, Hand and Reconstructive Microsurgery Cluster, National University Health System, Singapore.

${ }^{2}$ MOH Holdings, Singapore

Address for Correspondence: Dr Yu Han Chee, Department of Orthopaedic Surgery, National University Hospital Sports Centre, National University Health System, 1 East Kent Ridge Road, NUHS Tower Block, Level 11, Singapore 119074, Singapore

E-mail: yu_han_chee@nuhs.edu.sg 
1,300 in 1998 to 9,000 in 2050 , because of the aging population. ${ }^{1}$ Intertrochanteric fractures account for a significant number of these hip fractures. ${ }^{2}$

Several methods of fixation have been described over the years for intertrochanteric fractures of the hip. These include the use of dynamic hip screw, intramedullary nailing, and external fixation. ${ }^{3}$ Patients with significant comorbidities are often viewed as unsuitable candidates for general anaesthesia. As a result, their surgeries are often delayed or cancelled, resulting in prolonged suffering, pain, associated morbidity with immobilisation in bed, for example, pressure sores, pneumonia, urinary tract infection and, ultimately, early mortality.

External fixation was thus introduced several decades ago for the management of intertrochanteric fractures, but it fell out of favour owing to the high prevalence of postoperative complications, such as pin tract infections, pin-loosening and mechanical failure of the fixator. Various factors such as the advancement of external fixators in the last decade, introduction of new implants such as hydroxyapatite-coated pins, and better understanding of the mechanics of fracture healing, have prompted surgeons to reconsider external fixation as an alternative method for trochanteric fracture management. ${ }^{4}$

External fixation has since been described to have the ability to successfully treat high-risk patients with intertrochanteric fractures. It can be performed under local or regional anaesthesia, for example, lumbar plexus block, and confers shorter operative times with less blood loss. However, publications thus far have been limited to small case series ${ }^{5-17}$ and no published literature has attempted to analyse them in totality.

\section{Objective}

This current review aims to pool together these case series, and to evaluate the outcomes and complications of external fixation when performed in high-risk patients with intertrochanteric fractures.

\section{Materials and Methods}

The systematic review was performed using the Preferred Reporting Items for Systematic Reviews and Meta-Analyses (PRISMA) guidelines. The search was conducted using PubMed, Medical Literature Analysis and Retrieval System Online (MEDLINE), Cumulative Index to Nursing and Allied Health Literature (CINAHL) and The Cochrane Library through 1 March 2019. The keywords used were "external fixation OR internal fixation Or Dynamic Hip Screw" and "intertrochanteric* OR trochanteric* fracture* OR extracapsular*" and "high risk".

All studies that reported the outcomes of external fixation for high-risk patients with intertrochanteric fractures were included. Studies that were excluded from our series were those that involved nonintertrochanteric fractures, pathological fractures, patients who were not of the high-risk subgroup, intertrochanteric fractures that were not managed with external fixation, studies that did not report preoperative and postoperative outcomes, and studies where the outcomes cannot be retrieved. Review articles, non-English articles and articles with no full text availability were also excluded.

The articles were selected in 2 stages (Figure 1). In the first stage, the abstracts identified through the above searches were downloaded and screened using the inclusion and exclusion criteria. In the second stage, the full texts of the shortlisted articles fulfilling the criteria were downloaded and assessed for eligibility. The reference lists of the publications were then hand-searched for additional relevant studies. This process was repeated thrice independently.

Thereafter, each study's data was retrieved individually. The design for each study was retrieved and presented in Tables 1 and 2. The assessment of study design and risk of bias of each study are reflected in Tables 3 and 4. All clinical outcomes that were reported postoperatively were included in Table 5. These include perioperative details, radiological outcomes, clinical outcomes and complications.

\section{Results}

A total of 13 publications ${ }^{5-17}$ were included in the review. There were a total of 687 patients involving 687 hips. Six hundred and twenty-seven patients underwent external fixation for their intertrochanteric fractures, while 30 patients underwent internal fixation with a dynamic hip screw and another 30 patients underwent skeletal traction, as modalities of management in comparison with external fixation. The average age of the patients was 75.3 years (range: $12-100$ years). The mean follow-up duration was 13.7 months (range: 3-38 months). The average time of surgery for the patients was 24.1 minutes (range: 10-49 minutes) and the average hospital stay was 4.55 days (range: $0-45$ days). The average time to surgery was 3.21 days (range: 0-18 days). 


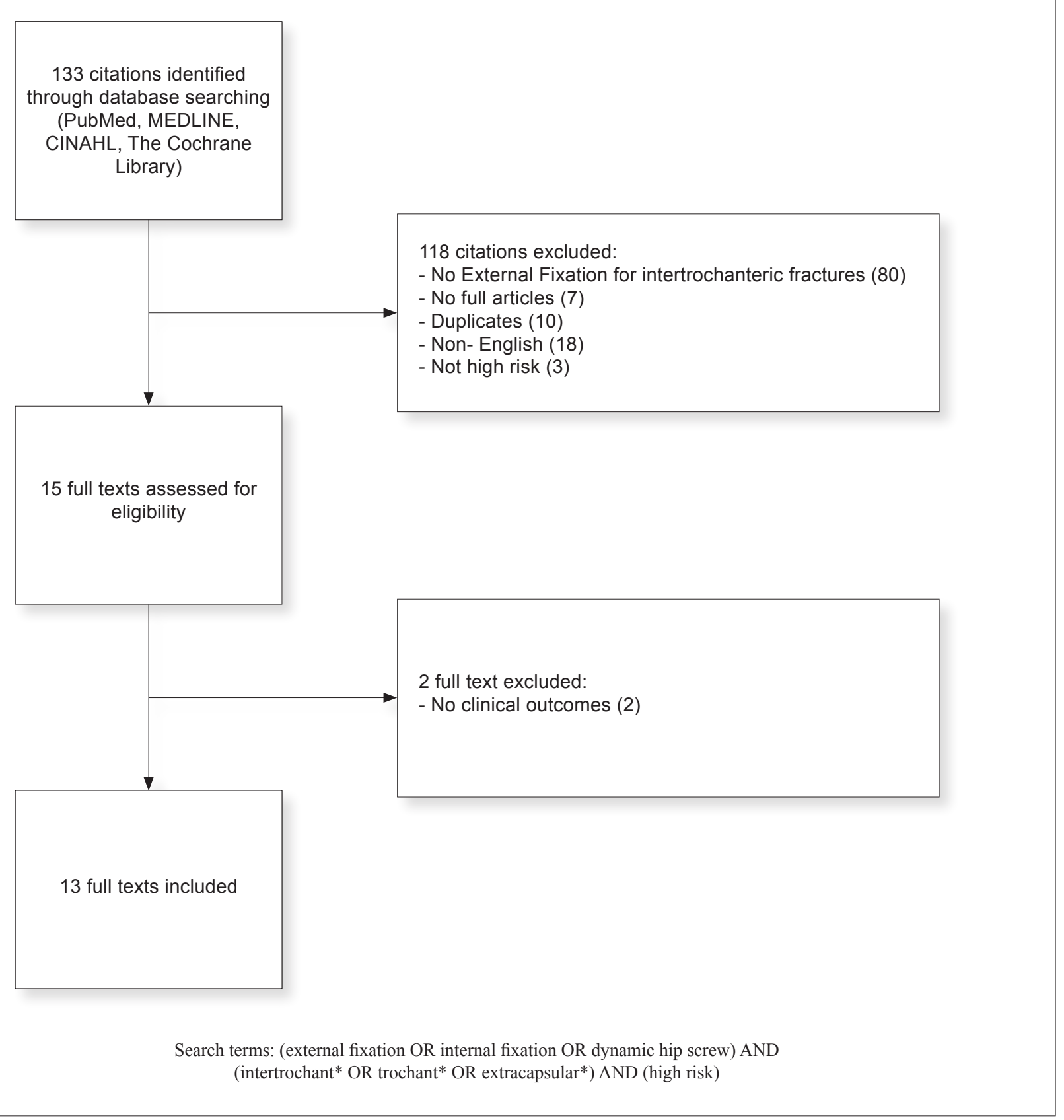

Fig. 1. Flow diagram of the review and selection of cases

\section{Radiological Outcomes}

Twelve of the studies reported radiological outcomes in terms of reduction of the fracture postoperatively, fracture healing or reduction of limb length discrepancy. All the studies noted acceptable reduction of the fracture postoperatively. Ten studies reported radiological outcome of varus angulation greater than $10^{\circ}$ postoperatively, and 7 studies reported the limb length discrepancy greater than $2 \mathrm{~cm}$ post fixation. Altogether, a total of 87 patients suffered from varus angulation of the limb that was greater than $10^{\circ}$ post fixation. Sixty-three patients suffered from persistent limb length discrepancy greater than $2 \mathrm{~cm}$ post fixation. 


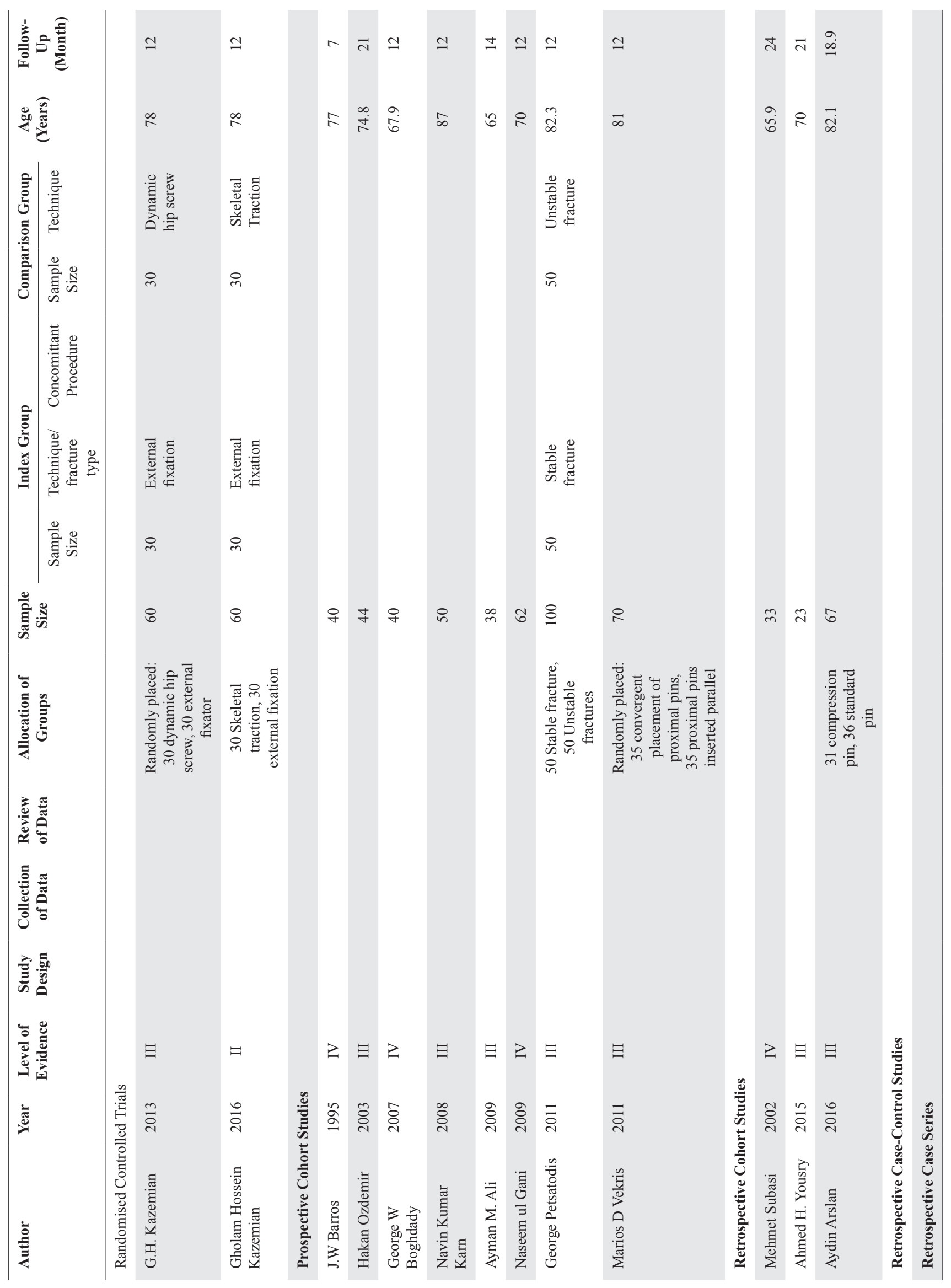




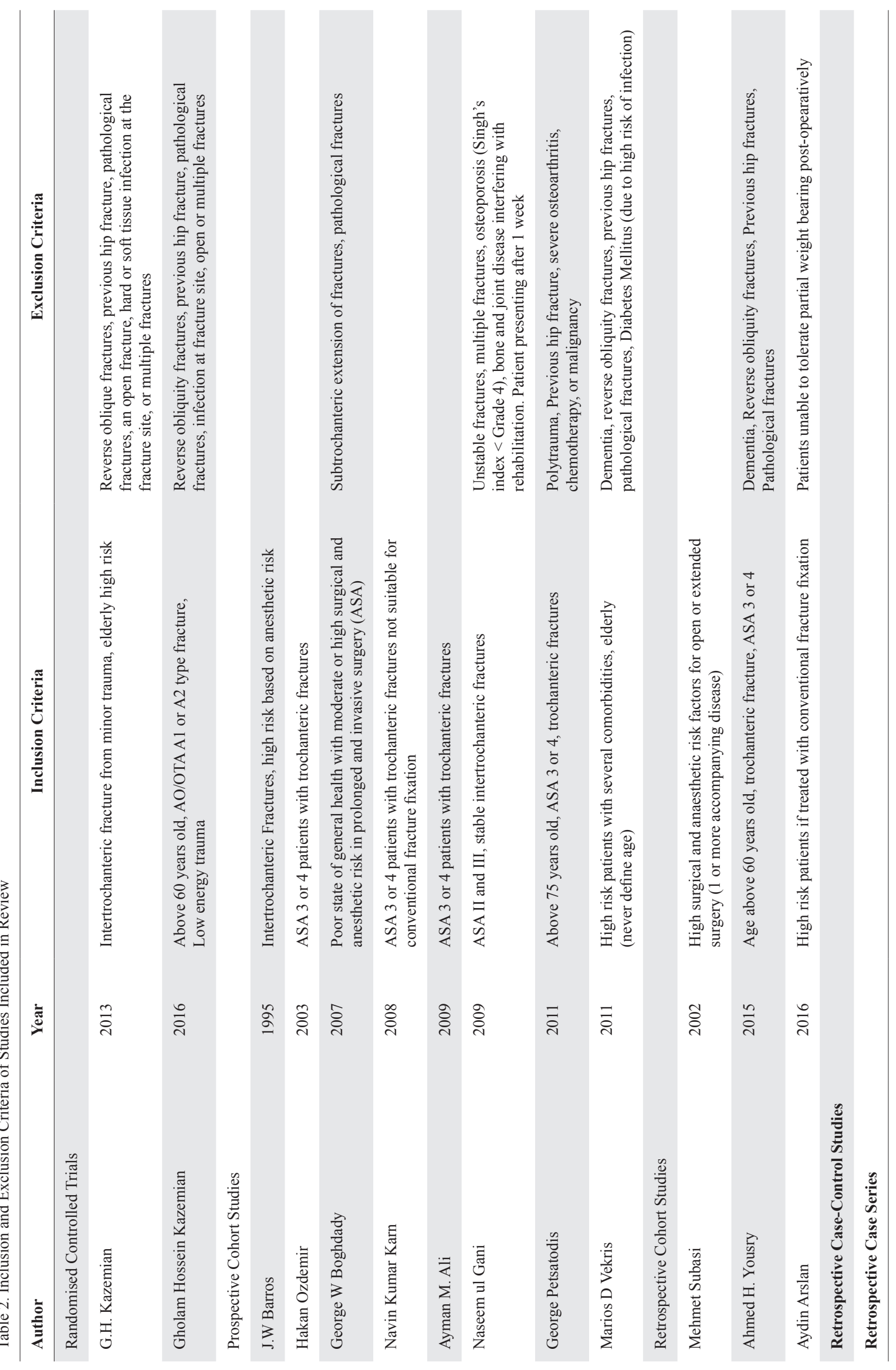




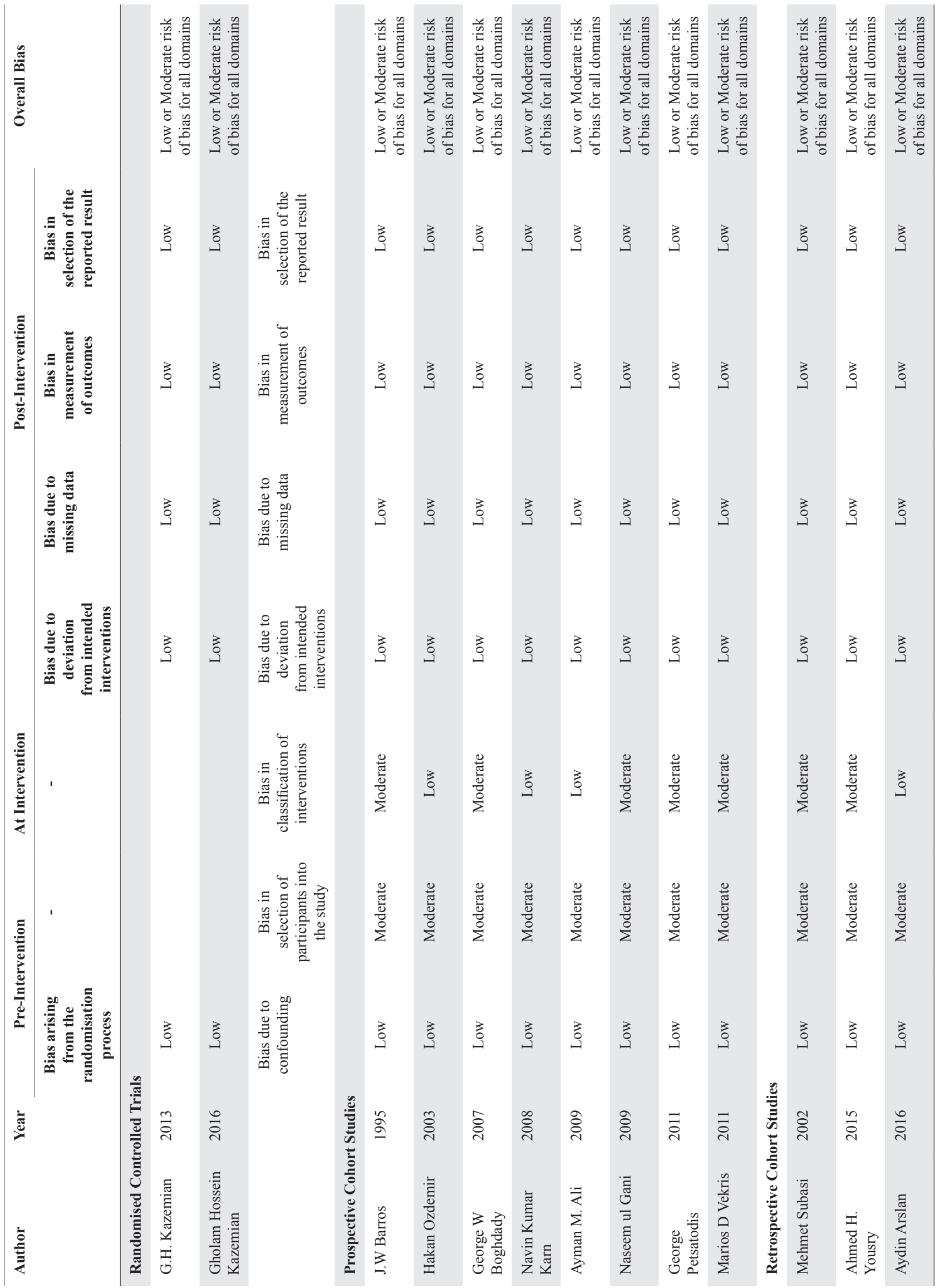




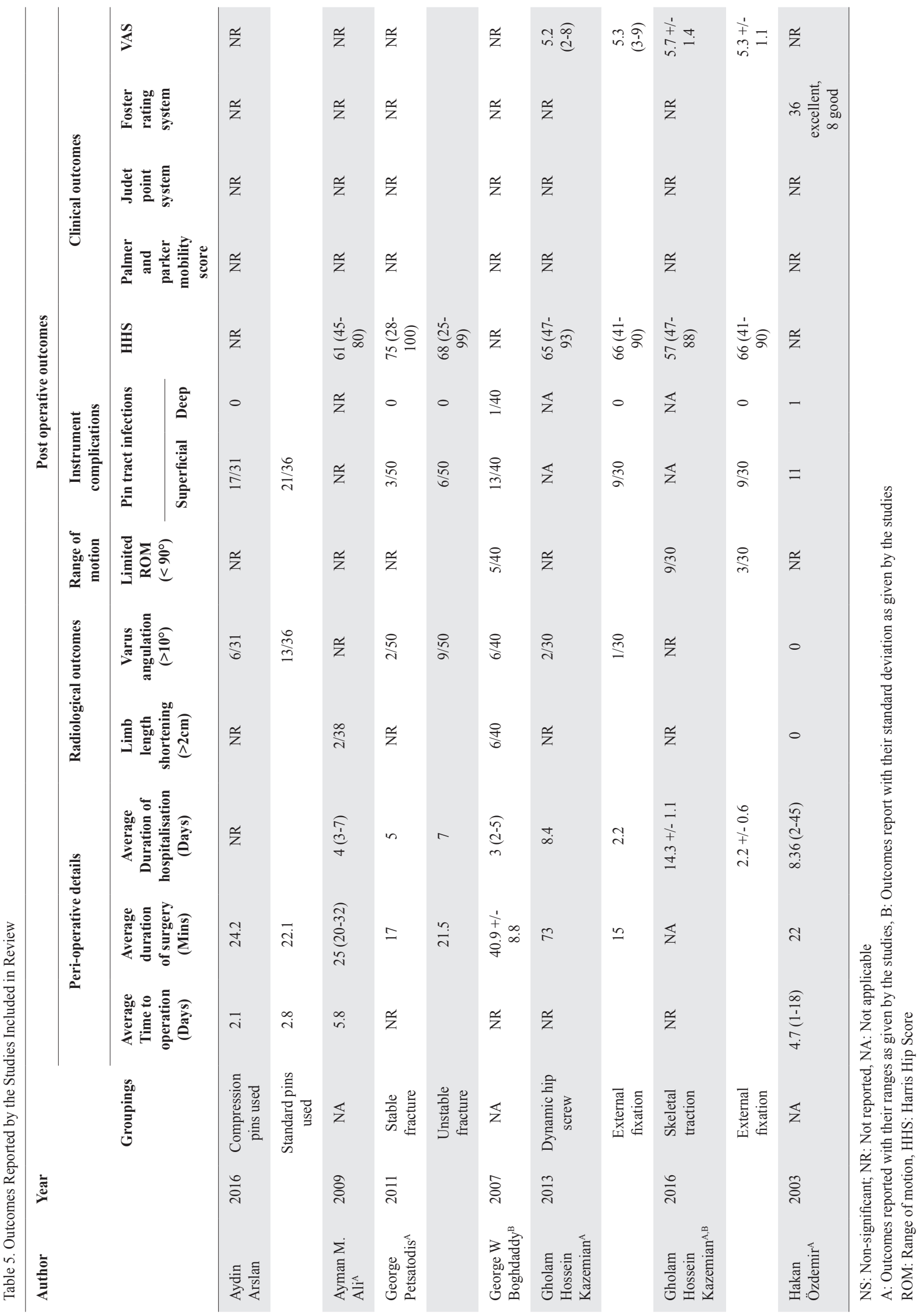




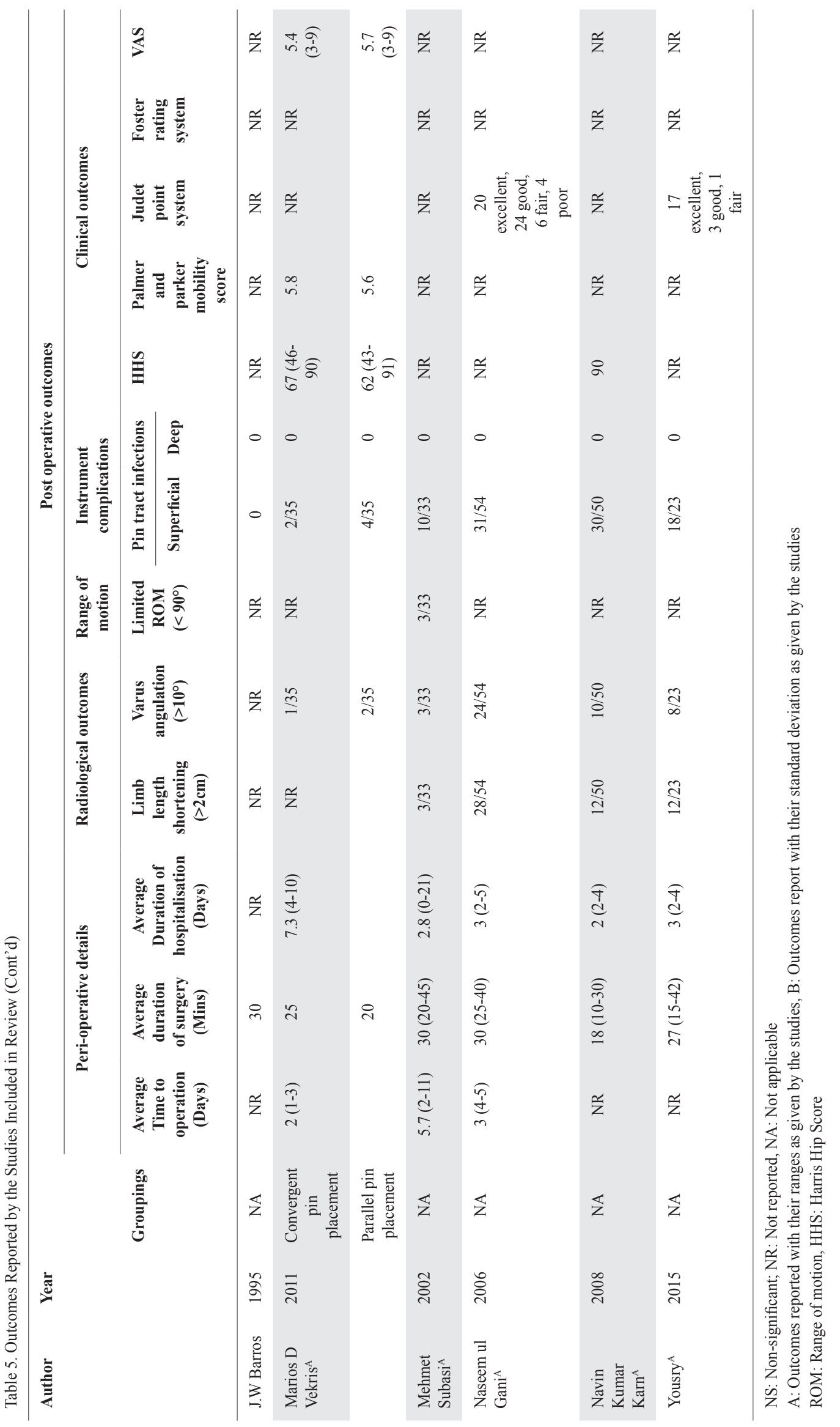




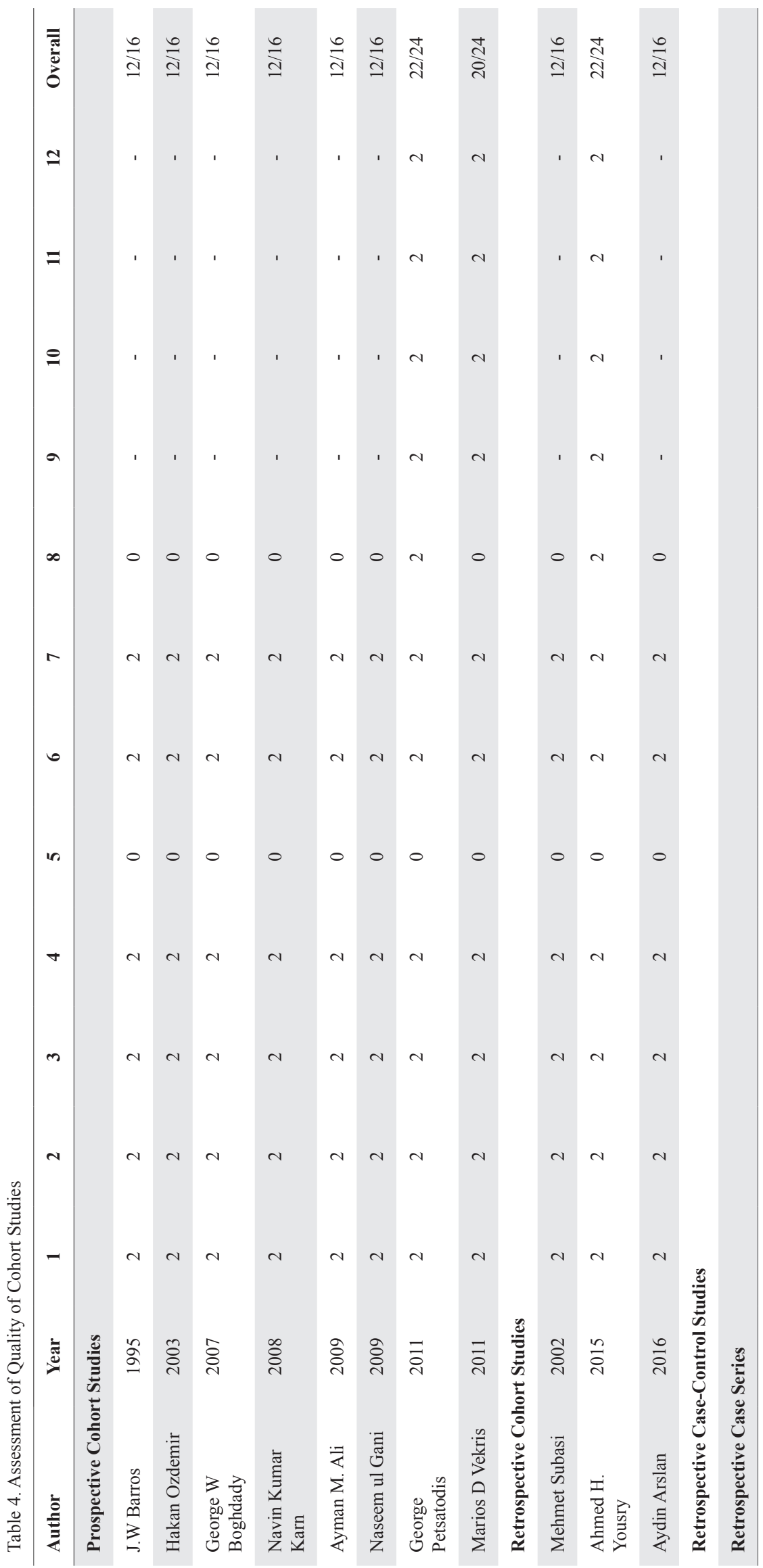




\section{Clinical Outcomes}

Twelve of the studies reported postoperative clinical outcomes (Table 5). Regarding clinical hip outcome scores, 6 studies reported outcomes based on Harris hip score, Palmer and Parker mobility score, Judet point system or Foster rating scale. Kazemian et al. ${ }^{7}$ noted better Harris hip scores after external fixation compared to skeletal traction and dynamic hip screw, respectively. Vekris et al. ${ }^{8}$ noted lower Harris hip scores and Palmer and Parker mobility scores for parallel screw placement in external fixation than in convergent screw placement in external fixation. The Harris hip score was lower after external fixation was done for unstable i ntertrochanteric fractures as compared to stable intertrochanteric fractures. ${ }^{9}$

Regarding other postoperative outcomes, 3 studies reported the visual analogue scale for pain. One of the studies noted higher pain scores for external fixation compared to dynamic hip screw, whereas another study noted lower pain scores for external fixation compared to skeletal traction. One of the studies noted lower pain scores for external fixation with parallel pin placement compared to convergent pin placement.

Four studies reported the outcome in terms of postoperative range of motion. All 4 studies noted limitation in the range of motion to less than $90^{\circ}$ post-operatively. Kazemian et al noted reduced occurrence of limitation in range of motion after external fixation compared to skeletal traction.

\section{Complications}

One patient who underwent external fixation suffered a spontaneous fracture of the ipsilateral neck of femur 3 weeks post fixation. The external fixator was removed and replaced with a hemiarthroplasty. ${ }^{11}$ Twenty-three patients in these studies suffered malunion. Fourteen patients suffered urinary tract infections, 8 patients had pneumonia, 7 patients had deep vein thrombosis, and 2 patients had pulmonary embolism. ${ }^{5-17}$ One hundred and three patients suffered from pin tract complications, such as pin tract infections, that were resolved with oral antibiotics. ${ }^{5-17}$ No case of osteomyelitis was noted, and no fixation washout had to be done. There was one case of deep pin tract infection that necessitated the removal of the pins.

\section{Discussion}

This study describes the promising and useful role of external fixation especially in the management of high-risk patients with intertrochanteric fractures. The procedure can help with radiological reduction of the fracture, reduction of limb length discrepancy, reduction of operative duration, decrease in postoperative immobility, reduction in pain and improvement in clinical outcome hip scores. It is versatile and seems able to accommodate both stable and unstable fractures.

External fixation was first described in 1995 by Barros et al as a suitable method of fixation for intertrochanteric fractures in high-risk patients. Subsequently, 12 other studies have been published regarding the use of external fixation in high-risk elderly patients. Of these studies, $2^{7,12}$ have compared external fixation to skeletal traction and dynamic hip screw, respectively. One study has compared the use of external fixation in both stable and unstable intertrochanteric fractures ${ }^{9}$ and 2 studies have compared 2 different techniques of external fixation for intertrochanteric fractures. ${ }^{8,10}$

Despite the increase in studies regarding the use of external fixation as an alternative for intertrochanteric fractures in high-risk patients, ${ }^{5-17}$ no published literature has analysed these studies in totality. Hence, this systemic review aims to pool together the studies to analyse the outcomes and complications of this procedure and compare it with other established treatment methods for intertrochanteric fractures.

External fixation confers the advantages of shorter operating time, shorter duration of hospitalisation, less blood loss, and lower anaesthetic risks compared to internal fixation, making it a more suitable option in managing high-risk patients. External fixation preserves the fracture haematoma, which is important for union. ${ }^{18}$

External fixation is commonly viewed as a bridging procedure before definitive management, such as internal fixation, is done, ${ }^{19}$ to allow for stabilisation of the patient and reduction in soft tissue swelling. In our review, it was evident that external fixation serves as a suitable alternative for definitive management in high-risk patients who are unable to tolerate a definitive fixation intraoperatively. Kazemian et al ${ }^{12}$ prospectively compared high-risk patients who underwent either dynamic hip screw or external fixation of intertrochanteric fractures, and found that there were no significant differences in comorbidities, quality of reduction and Harrison hip scores between the 2 groups. This further demonstrates the potential of external fixation to achieve equal or better outcomes compared to those of internal fixation in high-risk patients with intertrochanteric fractures.

An interesting finding in this review is that the fixation is versatile and is capable in treating both stable and unstable fractures. ${ }^{9}$ However, unstable fractures may 
be associated with higher postoperative morbidity, ${ }^{9}$ and it would be worthwhile to prognosticate based on the stability of the patient's fracture for better riskbenefit analysis preoperatively. Hence, the surgical team will be able to better counsel the family regarding the prognosis of the patient after treatment, despite external fixation showing the ability to successfully correct the deformities of unstable fractures postoperatively without open surgical intervention. Indeed, the greater morbidity of patients with unstable intertrochanteric fractures who undergo the fixation may be due to the fracture's inherent difficulty to achieve satisfactory reduction with union. ${ }^{9}$ Moreover, it is noted in previous studies that patients with unstable fractures also face higher morbidity than patients with stable fractures, even with other modalities of fixation such as dynamic hip screw and intra-medullary nailing. ${ }^{20,21}$

Another noteworthy finding is that the incorporation of a compression pin in the external fixation device has reduced the rates of complications compared to the use of standard pins solely. ${ }^{10}$ In the study done by Arslan et al, the compression pin allowed for the additional step of fracture compression and fragment stabilization intraoperatively. Patients who had a compression pin included were noted to have significantly lower rates of varus deformity and limb length discrepancy postoperatively. Indeed, it is known that compression helps to increase the stability of the external fixation frame, ${ }^{22}$ and greater surface area of contact between the fracture fragments allows for increased stability of the reduction. ${ }^{23}$ Hence, patients have the potential to benefit in terms of better functional outcomes if compression pins were utilised to stabilise the fracture fragments prior to fixation construction.

One significant complication noted in this review was that of pin tract complications. There is, at present, still little evidence-based recommendations on how to prevent pin tract infections, with different publications having different protocols on antibiotic prophylaxis and dressing changes. Recent literature on the prevention and treatment of pin tract infections reported lower adherence of staphylococcus and higher resistance of bacterial adhesion with the use of hydroxyapatite-coated coated pins. ${ }^{24}$ Another study also reported that patients who received $250 \mathrm{mg}$ cefazolin injections along the pin insertion site have statistically significant lower rates of pin tract infections. ${ }^{25}$ It is also thought that avoiding thermal necrosis when using power drills ${ }^{26}$ and implanting pins without excessive skin tension reduces pin site infection rates.
Surgeons are recommended to educate their patients on the signs and symptoms of pin tract infections so as to initiate treatment ${ }^{27}$ promptly. Only the study done by Barros et al did not have any pin tract complications noted. This may be because pins were removed definitively once reaction was seen around the pins, despite having no other clinical features of infection.

Another complication not uncommonly faced with the procedure is that of limb length discrepancy and varus angulation. Naseem et $\mathrm{al}^{6}$ and Yousry et $\mathrm{al}^{16}$ had a significant proportion (52\%) of patients who suffered from limb length discrepancy in their studies. However, these patients were pushed to ambulate earlier with the mean hospital stay being 3 days, that is, shorter than the other 4 studies that reported postoperative limb length discrepancy. In the study done by Naseem et $a l,{ }^{6}$ the high Judet point scores also showed that their patients were not functionally impaired despite the presence of limb length discrepancy post fixation. Similarly, patients in Yousry et al's study were noted to have satisfactory functional outcomes based on the Judet scale, with up to $95 \%$ of their cohort ${ }^{16}$ scoring excellent or good.

\section{Limitations}

While this systematic review is one of the first to analyse in totality the outcomes of high-risk patients treated with external fixation, it also faces several limitations. Firstly, the postoperative outcomes of the patients could not be measured in comparison with the preoperative outcomes, since most of the operations were done on an emergency basis. This leads to the inability of the study to directly compare external fixation with other procedures mentioned for intertrochanteric fractures. Secondly, the sample sizes in the studies under review were relatively small. This could be due to the fact that external fixation is rarely done for patients in most cases, unless they could not be optimised preoperatively due to various comorbidities. Thirdly, the power of this systematic review is limited by the lack of adequately powered studies in the current literature regarding external fixation for high-risk elderly patients. There might be publication bias as it is a widely accepted belief that external fixation is inferior to internal fixation in terms of outcomes, leading to fewer publications which favour internal fixation. However, with this review demonstrating the advantages of external fixation in this specific group of patients, more prospective and comparative studies can be done in the future to validate the outcomes shown. 


\section{Conclusion}

High-risk patients who are deemed unsafe to undergo general anaesthesia for internal fixation, and those where conservative treatment has failed usually because of persisting pain, can consider external fixation as an alternative treatment. External fixation confers radiological reduction of the fracture, enables a shortened operative duration, promotes fracture healing, reduces pain, restores limb length discrepancy, decreases postoperative immobility and improves clinical outcome hip scores. External fixation can be considered an equally efficacious management choice compared to internal fixation for this specific group of high-risk patients.

\section{REFERENCES}

1. Asia-Pacific Regional Audit. (2018).

2. Sonawane, Dhiraj. (2015). Classifications of Intertrochanteric fractures and their Clinical Importance. Trauma International. 1.7-11.

3. Socci, A. R., N. E. Casemyr, M. P. Leslie et. al. "Implant options for the treatment of intertrochanteric fractures of the hip: rationale, evidence, and recommendations." Bone Joint J. 2017 Jan; 99-B(1):128-133.

4. Vossinakis, I. C., and L. S. Badras. "The external fixator compared with the sliding hip screw for pertrochanteric fractures of the femur." J Bone Joint Surg Br 2002 Jan;84(1):23-9.

5. Barros, J. W., C. D. Ferreira, A. A. Freitas et al. "External fixation of intertrochanteric fractures of the femur." Int Orthop 1995; 19(4):217-9.

6. ul Gani, Naseem, Khursheed Ahmed Kangoo, Arshad Bashir et al. "External fixation of "intertrochanteric" fractures." Orthopedic reviews 1, no. 2 (2009).

7. Kazemian, Gholam Hossein, Mohammad Emami et al. "External fixation vs. skeletal traction for treatment of intertrochanteric fractures in the elderly." Trauma monthly 21, no. 1 (2016).

8. Vekris, Marios D., Marios G. Lykissas, Gregory Manoudis et al. "Proximal screws placement in intertrochanteric fractures treated with external fixation: comparison of two different techniques." Journal of orthopaedic surgery and research 6, no. 1 (2011): 48.

9. Petsatodis, George, Georgios Maliogas, John Karikis et al. "External fixation for stable and unstable intertrochanteric fractures in patients older than 75 years of age: a prospective comparative study.” J Orthop Trauma 2011 Apr;25(4):218-23.

10. Arslan, Aydın, Ali Utkan, and Tuba Tulay Koca. "Results of a compression pin along with trochanteric external fixation in management of high risk elderly intertrochanteric fractures." Indian J Orthop. Nov-Dec 2016; 50(6):636-40.

11. Boghdady, George W., and Mohammed Shalaby. "Safety and reliability of external fixation for basicervical and intertrochanteric fractures in high-risk elderly patients." Strategies in trauma and limb reconstruction 2, no. 2-3 (2007): 83-89.
12. Kazemian, Gholam-Hossein, Ali-Reza Manafi, Farideh Najafi et al. "Treatment of intertrochanteric fractures in elderly high-risk patients: dynamic hip screw vs. external fixation.” Injury 45, no. 3 (2014): 568-572.

13. Subasi, M., C. Kesemenli, A. Kapukaya et al. "Treatment of intertrochanteric fractures by external fixation." Acta Orthop Belg. 2001 Dec;67(5):468-474.

14. Özdemir, Hakan, T. Kürşat Dabak, Mustafa Ürgüden et al. «A different treatment modality for trochanteric fractures of the femur in surgical high-risk patients: a clinical study of 44 patients with 21-month follow-up.» Arch Orthop Trauma Surg. 2003 Dec; 123(10):538-543.

15. Ali, Ayman M., Mostafa Abdelkhalek, and Abdelrahman El-Ganiney. "External fixation of intertrochanteric fractures in elderly high-risk patients." Acta Orthop Belg. 2009 Dec;75(6):748-53.

16. Yousry, Ahmed H., Pranit N. Chotai, Sherif A. El Ghazaly et al. "Outcomes of trochanteric external fixation for geriatric inter-trochanteric hip fractures." J Orthop 2015 Jun 10;12(4):174-8.

17. Karn, Navin Kumar, Giris Kumar Singh et al. "Management of trochanteric fractures of the femur with external fixation in high-risk patients." International orthopaedics 33, no. 3 (2009): 785-788.

18. Schell, H., G. N. Duda, A. Peters, et al. "The haematoma and its role in bone healing." J Exp Orthop. 2017 Dec;4(1):5.

19. Della Rocca, G., \& Crist, B. (2006). External Fixation Versus Conversion to Intramedullary Nailing for Definitive Management of Closed Fractures of the Femoral and Tibial Shaft. Journal Of The American Academy Of Orthopaedic Surgeons, 14(Supplement), S131-S135. doi: 10.5435/00124635-200600001-00030

20. Knobe, Matthias, Gertraud Gradl, Andreas Ladenburger et al. "Unstable intertrochanteric femur fractures: is there a consensus on definition and treatment in Germany?." Clinical Orthopaedics and Related Research ${ }^{\circledR} 471$, no. 9 (2013): 2831-2840.

21. Setiobudi, Tony, Yau Hong Ng, Chin Tat Lim et al. "Clinical outcome following treatment of stable and unstable intertrochanteric fractures with dynamic hip screw." Ann Acad Med Singapore 2011;40(11):482.

22. Fragomen, A., \& Rozbruch, S. (2006). The Mechanics of External Fixation. HSS J. 2007 Feb;3(1):13-29. doi: 10.1007/s11420006-9025-0.

23. Podolsky A, Chao EY (1993) Mechanical performance of Ilizarov circular external fixators in comparison with other external fixators. Clin Orthop Relat Res. 1993 Aug;(293):61-70.

24. Arciola, Carla Renata, Lucio Montanaro, Antonio Moroni, et al. "Hydroxyapatite-coated orthopaedic screws as infection resistant materials: in vitro study." Biomaterials 20, no. 4 (1999): 323-327.

25. Nigam, Vishal, Anuj Jaiswal, and B. K. Dhaon. "Local antibiotics: panacea for long term skeletal traction." Injury 2005 Jan; 36(1):199-202.

26. Fragomen, Austin T., and S. Robert Rozbruch. "The mechanics of external fixation." HSS J $2007 \mathrm{Feb}$; 3(1):13-29.

27. Kazmers, Nikolas H., Austin T. Fragomen, and S. Robert Rozbruch. "Prevention of pin site infection in external fixation: a review of the literature." Strategies Trauma Limb Reconstr. 2016 Aug; 11(2):75-85. 